\title{
Avaliação da flexibilidade, equilíbrio e estado mental de idosas ativas e irregularmente ativas
}

\section{Evaluation of flexibility, balance and mental status of active and irregularly active elderly}

\section{Evaluación de la flexibilidad, equilibrio y estado mental de años activos e irregularmente activos}

\author{
Jénifer Carolina Fernandes (ㄴ) 1, Robson Ruiz Olivoto (1) 1 \\ Universidade Estadual do Oeste do Paraná (Unioeste). Marechal Cândido Rondon, PR, Brasil.
}

\begin{tabular}{|c|}
\hline $\begin{array}{l}\text { Article history } \\
\text { Received: 25/02/2019 } \\
\text { Accepted: } 26 / 04 / 2019 \\
\text { Published: } 29 / 08 / 2019\end{array}$ \\
\hline $\begin{array}{l}\text { Correspondent Author } \\
\text { Robson Ruiz Olivoto } \\
\text { Unioeste - Colegiado de Educação Física } \\
\text { Rua Pernambuco, } 1777 \text { - Cx. Postal } 91 \\
85960-000, \text { Marechal Candido Rondon, } \\
\text { PR, Brasil } \\
\text { robson.olivoto@unioeste.br }\end{array}$ \\
\hline
\end{tabular}

Editors

Alfredo Cataldo Neto

Newton Luiz Terra

Assistant Editors

Paula Engroff

Vanessa Sgnaolin

\begin{abstract}
RESUMO
OBJETIVO: Nosso objetivo foi avaliar os níveis de flexibilidade, equilíbrio e estado mental e comparar os resultados entre dois grupos, um composto por idosas ativas e outro por idosas irregularmente ativas.

MÉTODOS: A amostra foi composta por 24 idosas na faixa etária de 60 e 75 anos, divididas em dois grupos, Grupo de Idosas Ativas (GA) composto por idosas classificadas como ativas, e Grupo de Idosas Irregularmente Ativas (GI) composto por idosas classificadas como irregularmente ativas. Utilizamos o Questionário Internacional de Atividade física (IPAQ), e os testes de Sentar e Alcançar e escala de Equilíbrio de Berg e o Mini Exame do Estado Mental (MEEM). Nossos dados foram tratados através da estatística descritiva e comparativa, através do teste ANOVA one-way, com pós teste de Tukey, Correlação de Pearson e Teste $t$ de Student para amostras não pareadas. Os dados foram agrupados e apresentados como média e desvio padrão da média.

RESULTADOS: Nossos resultados não apresentaram correlação entre os níveis de flexibilidade e equilíbrio com o Mini Exame do Estado Mental. O GA apresentou melhores resultados nos testes de flexibilidade e equilíbrio quando comparados ao $\mathrm{Gl}$.

CONCLUSÃO: $\bigcirc$ GA apresentou resultados melhores nas variáveis flexibilidade e equilíbrio quando comparado ao $\mathrm{Gl}$ o que nos permite concluir que a prática regular de exercícios físicos pelo GA foi o fator determinante para que o grupo obtivesse melhores resultados que o Gl. No fator cognitivo, em nossa amostra, a prática de exercícios físico não foi um aspecto determinante.
\end{abstract}

DESCRITORES: Idosos; Exercício físico; Equilíbrio; Flexibilidade.

\footnotetext{
ABSTRACT

AIMS: Our objective was to compare the levels of effect of flexibility and balance beyond the cognitive aspect of two groups of active and irregularly active elderly women.

METHODS: The sample consisted of 24 elderly women in the age group of 60 and 75 years old, divided into two groups, Active elderly group (GA) composed of elderly women classified as active, and Group of irregularly active elderly (Gl) composed of elderly women classified as irregularly active. We used the International Physical Activity Questionnaire (IPAQ) and the Sit and Go and Berg Balance Scale tests and the Mental State Mini Exam (MSME). RESULTS: Our data were treated through descriptive and comparative statistics, through the ANOVA one-way test, with Tukey's posttest, Pearson's Correlation and Student's test for non-paired samples. The data were grouped and presented as mean and standard deviation of the mean. Our results showed no correlation between levels of flexibility and balance with the Mini Mental State Examination. The GA presented better results in the tests of flexibility and balance when compared to the Gl.

CONCLUSIONS: The GA presented better results in the variables flexibility and balance when compared to the GI, which allows us to conclude that the regular practice of physical exercises by GA was the determining factor for the group to obtain better results than the GI. In the cognitive factor, in our sample, physical exercise was not a determining factor.
}

KEYWORDS: Elderly; Physical exercise; Balance; Flexibility. 


\section{RESUMEN}

OBJETIVO: Nuestro objetivo fue evaluar los niveles de flexibilidad, equilibrio y estado mental y comparar los resultados entre dos grupos, uno compuesto por ancianas activas y otro por ancianas irregularmente activas.

MÉTODOS: La muestra fue compuesta por 24 ancianas en el grupo de edad de 60 y 75 años, divididas en dos grupos, grupo de ancianos activos (GA) compuesto por ancianas clasificadas como activas, y grupo de ancianos irregularmente activos (Gl) compuesto por ancianos ancianas clasificadas como irregularmente activas. Utilizamos el cuestionario internacional de actividad física (IPAQ), y las pruebas de sentar y alcanzar y escala de equilibrio de Berg y el mini examen del estado mental (MEEM). Nuestros datos fueron tratados a través de la estadística descriptiva y comparativa, a través de la prueba ANOVA de una forma, con post test de Tukey, correlación de Pearson y test t de Student para muestras no pareadas. Los datos fueron agrupados y presentados como media y desviación estándar de la media.

RESULTADOS: Nuestros resultados no presentaron correlación entre los niveles de flexibilidad y equilibrio con el mini examen del estado mental. El GA presentó mejores resultados en las pruebas de flexibilidad y equilibrio en comparación con el Gl.

CONCLUSIÓN: EI GA presentó resultados mejores en las variables flexibilidad y equilibrio cuando comparado al GI lo que nos permite concluir que la práctica regular de ejercicios físicos por el GA fue el factor determinante para que el grupo obtuviera mejores resultados que el Gl. En el factor cognitivo, en nuestra muestra, la práctica de ejercicios físicos no fue un aspecto determinante.

Palabras Clave: Ancianos; Ejercicio físico; Equilibrar; Flexibilidad.

\section{INTRODUÇÃO}

O processo de envelhecimento é um processo sequencial, individual, acumulativo e irreversível ${ }^{1}$, caracterizado por uma diminuição acentuada na capacidade funcional, o que de forma indireta aumenta a incidência de doenças associadas ${ }^{2}$. Conceitualmente pode-se definir capacidade funcional como sendo o conjunto associativo e harmônico entre saúde física, saúde mental, autonomia para as atividades de vida diária, integração familiar e social, além de independência econômica ${ }^{3}$, assim podemos perceber que a saúde física é um dos fatores, todavia o único ligado ao declínio biológico e com relação estreita com as variáveis da aptidão física.

O envelhecimento está associado a uma série de alterações na funcionalidade dos sistemas biológicos. Observa-se uma redução da massa magra (tecido muscular), redução na densidade e mineralização óssea fragilizando o corpo, podendo transformar o idoso em uma pessoa dependente até mesma para as mais simples atividades de vida diária

Neste sentido a prática regular de exercícios físicos pode ser uma ferramenta não farmacológica eficaz para combater este declino biológico característico do envelhecimento, melhorando, entre tantas, as funções cardiovasculares, melhora nas capacidades físicas como força em resposta as adaptações musculares mediadas pelo exercício, redução do processo de desmineralização óssea, que acabam por outras variáveis associadas a aptidão física ${ }^{5}$.

Durante o envelhecimento, as modificações mais evidentes são as que afetam diretamente o sistema anatomofuncional, com consequências na flexibilidade e força ${ }^{6}$. A flexibilidade é um elemento fundamental nas atividades de vida diária, o envelhecimento associado com o sedentarismo potencializa a redução de seus níveis?

Os processos degenerativos do sistema neuromuscular conduzem a redução na capacidade de desenvolver força (dinapenia), e perda de massa muscular (sarcopenia) $)^{5}$, que são os principais responsáveis pelo comprometimento da mobilidade, em especial na capacidade de manter-se em equilíbrio, aumentando efetivamente o risco de quedas e conduzindo a uma dependência funcional ${ }^{4}$. A redução nos níveis de equilíbrio corporal faz o idoso ser incapaz de controlar, de forma eficaz, seu centro de gravidade quer seja em situações estáticas ou em movimento, aumentando assim a sua instabilidade e risco a quedas ${ }^{8}$.

Além das alterações dos processos funcionais, ocorre também a alteração dos processos mentais no idoso, especialmente o déficit cognitivo, que nada mais é que a lentidão leve, geral e a perda da precisão, quando estes comparados com indivíduos mais jovens ${ }^{9}$.

Este conjunto de informações demonstram a importância da prática regular de exercícios físicos. Manter um estilo de vida ativo com prática de exercícios regularmente, promove um envelhecimento mais saudável e duradouro ${ }^{10}$.

Seguindo esta linha de raciocínio, podemos supor que idosos ativos fisicamente possuem uma vida mais saudável. Desta forma, nossa investigação tem como objetivo avaliar os níveis de flexibilidade, equilíbrio e estado mental e comparar os resultados entre dois grupos, um composto por idosas ativas e outro por idosas irregularmente ativas.

\section{MÉTODOS}

Esta pesquisa é do tipo quantitativa descritiva de caráter causal comparativa por apresentar como 
objetivo investigar um fenômeno a fim de esclarecê-lo sem modificar a sua realidade. Os dados Sociodemográficos da Assistência Social do município de Santa Helena-PR, indicam que 80 mulheres idosas residem no distrito de Moreninha, incluindo Vila (centro) e suas Linhas (zona rural), participantes e cadastradas no Grupo de Idosos do Município, com idade igual a 60 anos ou superior. Deste total, 45\% residem na Vila (centro) e 55\% residem na zona rural, respectivamente, 36 idosas e 44 idosas. Nossa amostra corresponde há $66 \%$ da população de mulheres idosas que residem na região denominada como Vila do distrito de Moreninha, Município de Santa Helena-PR

A amostra foi constituída por idosas, do gênero feminino, com faixa etária entre 60 e 75 anos de idade, moradoras no distrito de Moreninha, município de Santa Helena-PR, mais especificamente na Vila (centro). A amostra foi dividida em dois grupos: Grupo 1, denominado de Grupo de idosas Ativas (GA), foi composto por 12 idosas, classificadas como ativas, praticantes de caminhada e voleibol adaptado a pelo menos 3 meses no Grupo de Caminhada e Voleibol Adaptado dos Idosos de Moreninha. Este grupo participa de competições de voleibol para idosos e para tanto é submetido a um programa de exercícios físicos, adequados para a faixa etária e objetivos, com frequência de 3 sessões semanais; Grupo 2, denominado de Grupo de Idosas Irregularmente Ativas (GI), foi composto por 12 idosas, classificadas como irregularmente ativas, que não realizam nenhum exercício físico, mas realizam atividades físicas diárias, porém com intensidade insuficiente para serem classificadas como ativas.

A amostra do GA foi determinada com base no número de participantes do projeto de Caminhada e Voleibol Adaptado do Distrito de Moreninha, vinculado à Secretaria de Esportes do Município de Santa Helena, e que se enquadravam na faixa etária definida e que foram definidas como ativas através do questionário IPAQ. A partir da amostra do GA, definimos que o GI deveria contar com o mesmo número de participantes, dentro da mesma faixa etária. O GI foi selecionado por conveniência. Em virtude do tamanho do distrito, adotamos o procedimento de convite individual e pessoal, ou seja, nós fomos até as residências das idosas e fizemos o convite para participarem da coleta. No caso de a pessoa idosa aceitar participar e enquadrar-se nos critérios de inclusão, continuávamos os procedimentos de leitura, assinatura do TCLE e entrevista para o IPAQ. Se o resultado do IPAQ classificasse a pessoa idosa como sedentária ou irregularmente ativa ela seria convida a comparecer no local e horário que realizaríamos os testes.
Os participantes do estudo tiveram acesso e assinaram o Termo de Consentimento Livre e Esclarecido (TCLE). O projeto também foi analisado e aprovado pelo Comitê de Ética em Pesquisa, CAAE número 89484318.3.0000.0107.

Questionário Internacional de Atividade Física: A classificação das componentes de nossa amostra em ativas e irregularmente ativas foi dada a partir do Questionário Internacional de Atividade física (IPAQ), em sua versão longa, semana usual/normal, adaptada para idosos, por Benedetti, Antunes ${ }^{11}$. Mazo e Benedetti ${ }^{12}$, definiram, de forma mais objetiva, as características para interpretação dos 5 domínios e 15 questões do instrumento, ao acrescentarem estas informações que acabam explicando cada domínio:

1. Atividades Físicas no Trabalho - contempla 4 questões, com o objetivo de analisar o nível de atividade física durante o trabalho, e a intensidade das atividades.

2. Atividades Física como meio de Transporte composto por 3 questões, tem a finalidade de verificar como as idosas se deslocam de um lugar para outro;

3. Atividades Físicas em Casa: Tarefas Domésticas e Família - contempla 3 questões, tem a finalidade de investigar a atividade física que a idosa realiza dentro e ao redor de sua casa.

4. Atividades Físicas de Recreação, Esporte, Exercício Físico e de Lazer - são 3 questões ao todo, que correspondem as atividades físicas que a idosa faz relacionadas a recreação, esporte, exercício e lazer.

5. Tempo Gasto Sentado - contempla 2 questões, compete ao tempo em que a idosa gasta sentado no grupo de convivência, em casa, na igreja, trabalho, tempo livre, atividades manuais, visitando amigos e parentes e consultório médico.

Assim as idosas classificadas como ativos foram as que atingiram os seguintes escores: a) Vigorosa: $\geq 3$ dias/sem e $\geq 20$ minutos por sessão; b) Moderada ou Caminhada: $\geq 5$ dias/sem e $\geq 30$ minutos por sessão; c) Qualquer atividade somada: $\geq 5$ dias $/$ sem e $\geq 150$ minutos/sem (caminhada + moderada + vigorosa); já as idosas classificadas como irregularmente ativas foram as que não atingir os escores de ativas quando responderam o IPAQ ${ }^{11}$.

Teste de flexibilidade: $\mathrm{O}$ teste de "Sentar e Alcançar" foi proposto inicialmente por Wells e Dillon ${ }^{13}$, adaptado e utilizado para uma grande população por Guedes e Guedes ${ }^{14}$ e Guedes e Barbanti ${ }^{15}$, posteriormente atingindo outras clientelas, como nas 
pesquisas de Achour Júnior ${ }^{16}$ e Gorla ${ }^{17}$ que usou o teste com populações especiais, entre elas de idosos. O teste é realizado com auxílio de um instrumento composto por um caixote de madeira com superfície de 56,5 centímetros de cumprimento e 30,5 centímetros de aresta, com objetivo de determinar níveis de flexibilidade. Na parte superior da caixa é fixada uma escala de medida de $50 \mathrm{~cm}$ e os pés do avaliado ficam apoiados contra o caixote a $23 \mathrm{~cm}^{18}$. A classificação ou escores para determinar os níveis de flexibilidade estão descritos na Tabela 1.

Tabela 1. Classificação para o Teste de Sentar e Alcançar para mulheres entre 60 e 69 anos, e acima de 70 anos de idade.

\begin{tabular}{cccccc}
\hline Idade & Ótimo & Bom & Médio & Regular & Fraco \\
$60-69$ & $>34$ & $28,4-34$ & $22-28,4$ & $16-22$ & $<16$ \\
$70 \mathrm{ou}+$ & $>32$ & $22,5-32$ & $20-25$ & $15-20$ & $<15$ \\
\hline
\end{tabular}

Fonte: Albino et al. (2010) ${ }^{32}$.

Teste de equilíbrio: A Escala de Equilíbrio de Berg (anexo III), é um instrumento proposto por Kathy Berg, que avalia o desempenho normal do equilíbrio em tarefas motoras. A realização do teste dura aproximadamente 15 minutos, é composta por 14 itens, desenvolvidos em diferentes bases de apoio em tarefas físicas gerais ${ }^{19}$. A pontuação máxima que pode ser obtida é 56 pontos.

A Escala de Equilíbrio de Berg, no Brasil, foi validada por Woellner, Araujo ${ }^{19}$. Os autores realizarão uma correlação de coeficiente inter-observadores 0,98 $(\mathrm{p}<0,001)$ e intra-observadores de 0,99 ( $\mathrm{p}<0,001)$. Os resultados indicaram confiabilidade nos resultados. Todos os procedimentos descritos por Woellner, Araujo ${ }^{19}$ foram seguidos durante a realização do teste.

\section{Avaliação do Estado Mental}

O Mini Exame de Estado Mental (MEEM) (anexo IV e V), é o teste para avaliar o estado mental mais utilizado por tratar-se de um questionário de rápida e fácil aplicação. Utilizamos o teste para determinar as questões que envolvem os aspectos mentais de nossa amostra. É um instrumento com objetivo de avaliar funções cognitivas específicas, composto por questões agrupadas em sete categorias: orientação temporal (5 pontos); orientação espacial (5 pontos); registro de três palavras, avaliando a memória de curto prazo (3 pontos); atenção e cálculo (5 pontos); recordação das três palavras, avaliando a evocação (3 pontos); linguagem (8 pontos); e capacidade Visio construtiva (1 ponto). A pontuação varia de 0 a 30 pontos, sendo os valores mais baixos indicativos para déficit cognitivo e os mais altos, acima de 26 pontos, correspondentes a uma ótima capacidade cognitiva ${ }^{20,21}$.

A sequência organizacional para aplicação dos testes levou em consideração a praticidade de realização. O teste de flexibilidade, em função de sua praticidade de execução, foi o primeiro a ser realizado; para evitar o contato entre as idosas após realizarem o teste de MEEM, deixamos este teste para ser o último a ser aplicado e a aplicação foi organizada para que as pessoas que respondiam o questionário não tivessem contato com as que ainda não haviam respondido. Este procedimento foi adotado para minimizar os erros na coleta, ou seja, a troca de informações entre os participantes; em função desta organização, inicial e final, aplicamos o teste de Equilíbrio de Berg entre os testes já agendados. Todos os dados foram coletados pelo mesmo avaliador afim de minimizar a possibilidade de erro durante as coletas.

Os dados foram tratados através da estatística descritiva. Os resultados foram apresentados como média e desvio padrão da média. Para determinar se existia diferença entre as médias das três variáveis analisadas, utilizamos o teste ANOVA one-way, com pós teste de Tukey. No caso de apresentar diferença estatística utilizamos o Teste $t$ de Student para amostras independentes, para confirmar a diferença este as médias dos testes realizados. Para determinar se houve ou não correlação entre os testes, utilizamos a correlação de Pearson. O tratamento estatístico dos dados foi realizado através do software GraphPad Prism.

\section{RESULTADOS}

A amostra compreendeu 24 idosas divididas em dois grupos, GA e GI. Os dados da caracterização de nossa amostra são apresentados na Tabela 1. A média de idade do GA foi de 67,2 $\pm 4,51$ anos, enquanto o GI foi de $67,5 \pm 4,5$ anos, com idades máximas e mínimas de, respectivamente, 74 e 60 anos de idade, em ambos os grupos. A média de idade das amostras não apresenta diferença estatística, indicando ser uma amostra homogênea.

As participantes do estudo foram classificadas como ativas e irregularmente ativas antes de fazerem parte da amostra do estudo. Esta classificação foi determinada a partir do questionário IPAQ na sua versão completa e adaptada para idosos. A partir dos resultados, agrupamos nossa amostra nos grupos GA (ativo) e GI (irregularmente ativo). A Tabela 2 apresenta os dados quantitativos obtidos a partir do questionário IPAQ, organizamos a média de cada grupo, a partir da relação com frequência e duração em cada tipo de intensidade proposta pelo questionário. 
Tabela 2. Dados quantitativos do Questionário Internacional de Atividade Física (IPAQ) expressos em tempo (dias por semana/minutos contínuos). Dados organizados em média.

\begin{tabular}{|c|c|c|c|c|c|c|c|}
\hline & \multicolumn{2}{|c|}{ Caminhada } & \multicolumn{2}{|c|}{ Moderada } & \multicolumn{2}{|c|}{ Vigorosa } & \multirow{2}{*}{ Classificação } \\
\hline & Frequência & Duração & Frequência & Duração & Frequência & Duração & \\
\hline GA & 4,67 & 30,83 & 5,67 & 72,5 & 1,08 & 38,33 & Ativas \\
\hline GI & 2,08 & 17,08 & 3,17 & 22,92 & 0,17 & 3,33 & Irregularmente ativas \\
\hline
\end{tabular}

Fonte: Dados dos autores.

Nossos dados foram tratados, inicialmente, através do teste ANOVA one-way, com pós-teste de Tukey's. $O$ resultado do teste indicou diferença estatística entre as variáveis equilíbrio $(\mathrm{p}=0,0053)$ e flexibilidade $(\mathrm{p}=0,044)$ quando comparados os dados do GA com o GI, mas não apresentou diferença na variável MEEM $(\mathrm{p}=0,456)$ entre os grupos. Da mesma forma o teste de correlação de Pearson também não apresentou correlação entre a variável MEEM e as demais variáveis investigadas.

Nossos resultados do Mini Exame de Estado Mental, indicam que não há diferença estatística entre a amostra GA e GI $(p=0,456)$. Tais dados sugerem que o estado cognitivo, em especial nesta faixa etária, ou seja, em idosas, não tem relação com a prática ou ausência da prática de exercício físico (Tabela 5).

Tabela 3. Dados quantitativos do teste de Sentar e Alcançar (flexibilidade) expressos em centímetros. Dados organizados em média e desvio padrão da média.

\begin{tabular}{lcccc}
\hline Amostra & N & Flexibilidade & DP & Valor de $\mathbf{p}$ \\
GA & 12 & 27,2 & $\mathbf{\pm 8 , 5 2}$ & 0,044 \\
GI & 12 & 18,7 & $\mathbf{1 0 , 7 0}$ & \\
Total & 24 & & & \\
\hline
\end{tabular}

Fonte: Dados dos autores.

Tabela 4. Dados quantitativos do teste de equilíbrio de Berg, dados expressos na forma de scores. Dados organizados em média e desvio padrão da média.

\begin{tabular}{lcccc}
\hline Amostra & N & Equilíbrio & DP & Valor de $\mathbf{p}$ \\
GA & 12 & 55,8 & $\mathbf{\pm 0 , 4 5}$ & 0,0053 \\
GI & 12 & 52,3 & $\mathbf{\pm 3 , 8 9}$ & \\
Total & 24 & & & \\
\hline
\end{tabular}

Fonte: Dados dos autores.

Tabela 5. Dados quantitativos gerais do Mini Exame de Estado Mental (MEEM) expresso em scores. Dados organizados em média e desvio padrão da média.

\begin{tabular}{ccccc}
\hline Amostra & N & MEEM & DP & Valor de p \\
GA & 12 & 25,8 & $\mathbf{\pm 3 , 0 1}$ & 0,456 \\
GI & 12 & 24,9 & $\mathbf{2 , 9 1}$ & \\
Total & 24 & & & \\
\hline
\end{tabular}

Fonte: Dados dos autores.

\section{DISCUSSÃO}

Nossos resultados indicam que o grupo de idosas praticantes regular de exercícios físicos apresentam melhora nos índices de flexibilidade e equilíbrio quando comparados aos do grupo de idosas que não praticam exercícios físicos. De forma geral, a prática de exercícios físicos pode ser um fator determinante na redução dos declínios que estão associados ao envelhecimento ${ }^{22}$.

O desenvolvimento ou ampliação nos níveis das variáveis da aptidão física, em especial flexibilidade e equilíbrio (força muscular e controle motor) são de extrema importância quando se trata de qualidade de vida e autonomia para pessoas idosas ${ }^{22-24}$. Um programa regular, sistematizado e organizado de exercícios físicos regulares pode promover melhoras das variáveis da aptidão física funcional, dentre elas a flexibilidade, força, equilíbrio, dentre outras, que atuam efetivamente na melhora da qualidade de vida do idoso ${ }^{5}$.

A flexibilidade é uma variável motora importante associada a qualidade de vida em idosos ${ }^{23}$, entretanto ao longo do envelhecimento sofre modificação importantes que conduzem as alterações no desempenho em atividades da vida diária, mais especificamente, um decréscimo na qualidade de realização destas atividades $^{7}$. A prática regular de exercícios físicos podem ser uma ferramenta importante para controlar esta redução nos níveis de flexibilidade, tanto em populações adultas quanto em idosos ${ }^{6}$.

O GA de nosso estudo apresentou melhores índices de flexibilidade quando comparado aos do GI. Estes resultados podem ser consequência da ausência da prática de exercícios físicos pelo GI. O encurtamento muscular ou até mesmo a atrofia são fenômenos característicos da ausência de exercícios físicos, e manifestam-se mais intensidade quando se trata de idosos $^{25}$.

O envelhecimento traz consigo modificações funcionais importantes, a diminuição dos níveis de flexibilidade normalmente está associada a redução na capacidade de realizar força ${ }^{6}$, que em conjunto afetam diretamente a realização das atividades diárias ${ }^{25}$. A prática regular de exercícios, dentre estes o treinamento resistido, pode ser um fator que contribua efetivamente 
para a melhora da flexibilidade e variáveis associadas, como força e controle motor ${ }^{5,25}$.

O equilíbrio é outro componente da aptidão física que sofre influência direta do envelhecimento. A capacidade de manter-se equilibrado ou ter controle do equilíbrio corporal necessita que muitos sistemas biológicos atuem em conjunto. O sistema vestibular é responsável por organizar a orientação espacial, quer seja em situação estática ou dinâmica; o sistema proprioceptivo, através de seus proprioceptores responsáveis pela identificação dos momentos dos segmentos corpóreos; o sistema visual, possui relação significativa com o equilíbrio, ou seja, todos sistemas pertencentes ao Sistema Nervoso Central (SNC) e Sistema Nervoso Periférico (SNP) ${ }^{26}$.

A população idosa apresenta uma redução sensível nesta capacidade condicionante a ponto de limitar a capacidade de coordenação de movimentos motores ${ }^{4}$. Matsudo, Matsudo ${ }^{23}$ já associava a redução na capacidade de equilíbrio como sendo uma causa efetiva em acidentes oriundos de quedas. Em conjuntos estes autores indicam ser, esta, uma variável importante quando se trata de qualidade de vida em idosos.

O envelhecimento, de forma geral, é a degeneração funcional dos sistemas biológicos, um desgaste funcional dos sistemas, e todos os sistemas mecanismos tem controle regulado por centros superiores, ou seja, SNC. Neste sentido, a prática regular de exercícios físicos, contribui, efetivamente, na manutenção funcional dos sistemas biológicos, e contribui assim para manutenção de níveis adequados de aptidão física, dentre eles a flexibilidade e força que estão diretamente associados ao equilíbrio corporal ${ }^{5,24}$.

Apesar da atividade física estar relacionada com melhora em variáveis neuromotoras, força muscular, flexibilidade, aumento do gasto energético acima dos níveis de repouso, dentre outras ${ }^{23,27}$, nosso GI, que está envolvido ativamente em atividade agrícolas (exercício não sistematizada, ou seja, atividade física) não apresenta resultados significativos nas variáveis da aptidão física investigadas, indicando, que, do ponto de vista de saúde, para idosos, as adaptações necessárias são oriundas da prática regular de exercícios físicos e de uma mudança comportamental seguida de uma continuidade desta prática².

Além do declínio nas funções motoras, há evidências de que o envelhecimento também promova o mesmo declínio nas funções cognitivas ${ }^{20}$. Como o declínio nas funções motoras pode ser minimizado pela prática regular de exercícios físicos, como demonstrado pelos autores já citados, e em especial pelos dados por nós apresentados, surgiu a necessidade de se investigar se os resultados benéficos da prática regular de exercícios físicos podem ser extrapolados para as funções cognitivas.

O instrumento MEEM é utilizado regularmente para avaliar a função cognitiva e o rastreamento de estágios demenciais. A confiabilidade do instrumento foi testada relacionando-a com características sociodemográficas, que demonstraram se um instrumento com boa consistência interna e comprovada confiabilidade, entretanto sofre influência de variáveis como gênero e faixa etária ${ }^{28}$. Em nosso estudo controlamos estas variáveis, uma vez que o gênero e faixa etária de nossos grupos é o mesmo.

Nossos resultados não apresentaram correlação entre os resultados das variáveis de flexibilidade e equilíbrio com os resultados do MEEM. Nos estudos conduzidos por Lira e Santos ${ }^{29}$ que investigaram a função cognitiva (avaliada através do MEEM) e capacidade funcional em idosos portadores de Alzheimer, foi observada correlação entre os resultados do MEEM e capacidade funcional, todavia, a capacidade funcional foi determinada a partir de um teste de levantar da cadeira denominado de TUG (time up and go test). Estes resultados são diferentes dos encontrados em nosso estudo, o que pode ser explicado pelo protocolo para determinação da capacidade funcional e da amostra serem diferentes dos adotados em nosso estudo.

Satler, Diniz ${ }^{30}$ investigaram se o nível educacional e idade poderiam interferir no resultado do MEEM em idosos. Seus dados indicaram haver uma relação direta entre nível educacional e resultado no MEEM, ou seja, quanto maior fosse a escolaridade melhor seria o desempenho no MEEM. Nossos resultados não apresentam a mesma relação descrita pelos autores. $\mathrm{O}$ GI apresenta escolaridade média de 4,5 anos, enquanto o grupo GA, média de 3,9 anos de estudo. Apesar do grau de escolaridade do GI ser maior, o GA apresentou resultados médios maiores no MEEM, todavia os resultados não apresentaram diferença estatística, sugerindo que a variável estado mental, nesta amostra, não tem relação com o nível educacional.

Dias, Streit ${ }^{20}$ investigou os aspectos cognitivos entre idosos praticantes e não praticantes de exercício físico. Dividiu seus grupos em G1 que praticava exercícios físicos e G2 que não praticava. Quando comparamos os resultados médios do MEEM entre os estudos percebemos que os dados são similares, estatisticamente iguais. $\mathrm{O}$ GA e $\mathrm{G} 1$ tiveram as seguintes médias no MEEM, respectivamente, $25,8 \pm 3$ e $25,4 \pm 1,6$ e o GI e G2 tiveram, respectivamente, as seguintes médias, $24,9 \pm 2,9$ e $25,4 \pm 1,4$. Embora tratamos nesse estudo que a prática regular de exercícios físicos pode contribuir para uma melhora na capacidade mental/cognitiva, nossos resultados não confirmaram tal hipótese. 
Apenas de nossos dados não apresentarem uma relação, a prática regular de exercícios físicos ou atividades físicas, pode influenciar diretamente na melhora da qualidade de $v^{2} \operatorname{lda}^{23}$ e das capacidades funcionais do idoso ${ }^{6}$. Aumentando a qualidade de vida, também melhorando a autoestima, bem-estar, sensações de autoeficácia, reduzindo riscos de depressão e ansiedade ${ }^{31}$. Dessa forma, não apenas o exercício, mas também o trabalho constante das funções cognitivas, como hábito de leitura, estimulação para a educação, trabalho da memória através de atividades diárias, busca por informações em redes sociais, internet e etc, podem ser fatores que venham a contribuir para a melhora do estado cognitivo, mas tal afirmação somente poderá ser feita com uma nova e mais aprofundada investigação.

\section{CONCLUSÃO}

Neste sentido, concluímos com este estudo que as idosas fisicamente ativas possuem resultados melhores nas capacidades de flexibilidade, equilíbrio, quando comparadas as idosas irregularmente ativas. Ressaltando a importância da prática regular de exercícios físicos em todas as fases da vida, principalmente na velhice, onde ocorre o declínio especialmente das funções físicas. Nosso estudo apresenta limitações em especial no tamanho da amostra. Apesar de ser representativa em relação a população onde estão inseridas, o número pode ser considerado pequeno.

É fundamental que novos estudos, nestes mesmos moldes, sejam realizados, com amostras das mais variadas regiões do país a fim de fornecer dados importantes sobre, não só o estado de envelhecimento populacional, mais principalmente, se os idosos estão praticando exercícios, se existem políticas públicas que fomentem esta prática e especialmente, resultados que confirmem a grande importância que o exercício físico tem na manutenção da saúde, em especial para essa clientela.

\section{REFERÊNCIAS}

1. Brandalize D, de Almeida PHF, Machado J, Endrigo R, Chodur A, Israel VL. Efeitos de diferentes programas de exercícios físicos na marcha de idosos saudáveis: uma revisão. Fisioter Mov. 2011;24(3):549-56. https://doi. org/10.1590/s0103-51502011000300019

2. Scherer FC, Gerage AM, Borges LJ, Borges RA, Melo FC, Teixeira DC, Benedetti TRB. Efeito de dois programas na promoção da atividade física em idosos. RBAFS. 2018;23:e0034. https://doi.org/10.12820/rbafs.23e0034

3. Brito TA, Fernandes MH, Coqueiro RS, Jesus CS, Freitas R. Capacidade funcional e fatores associados em idosos longevos residentes em comunidade: estudo populacional no Nordeste do Brasil. Fisioter Pesqui. 2014;21(4):308-13. https://doi.org/10.1590/s0102-311x2008000200020
4. Agostini CM, Rodrigues VS, Guimarães AC, Damázio LCM, Vasconcelos NN. Análise do desempenho motor e do equilíbrio corporal de idosos ativos com hipertensão arterial e diabetes tipo 2. Rev Aten Saúde. 2018;16(55):29-35. https://doi.org/10.13037/ ras.vol16n 55.4690

5. Fonseca AIS, Barbossa TC, Silva BKR, Ribeiro HS, Quaresma FRP, Maciel ES. Efeito de um programa de treinamento de força na aptidão física funcional e composição corporal de idosos praticantes de musculação. RBPFEX. 2018;12(76):556-63. https://doi.org/10.17058/ cinergis.v16i3.6528

6. Moura ES, Castro HO, Aguiar SS, Borges Júnior M, Costa GCT, Pires FO, Soares KVBC. Avaliação do nível de força e flexibilidade de idosos praticantes de atividades físicas. RBPFEX. 2018;12(75):496-502.

7. Silva NA, Menezes TN. Capacidade funcional e sua associação com idade e sexo em uma população idosa. Rev Bras Cineantropom Desempenho Hum. 2014;16(3): 359-70. https://doi.org/10.5007/1980-0037.2014v16n3p359

8. Coutinho ESF, Silva SD. Uso de medicamentos como fator de risco para fratura grave decorrente de queda em idosos. Cad Saúde Pública. 2002;18(5):1359-66. https:// doi.org/10.1590/s0102-311x2002000500029

9. Ferreira PCS, Tavares DMS, Rodrigues RAP.

Características sociodemográficas, capacidade funcional e morbidades entre idosos com e sem declínio cognitivo. Acta Paul Enferm. 2011;24(1):29-35. https://doi. org/10.1590/s0103-21002011000100004

10. Ribeiro F, Gomes S, Teixeira F, Brochado G, Oliveira J. Impacto da prática regular de exercício físico no equilíbrio, mobilidade funcional e risco de queda em idosos institucionalizados. Rev Port Ciên Desp. 2009;9(1): 36-42. https://doi.org/10.5628/rpcd.09.01.36

11. Benedetti TRB, Antunes PC, Rodriguez-Añez CR, Mazo GZ, Petroski EL. Reprodutibilidade e validade do Questionário Internacional de Atividade Física (IPAQ) em homens idosos. Rev Bras Med Esporte. 2007;13(1): 11-6. https://doi.org/10.1590/s1517-86922007000100004

12. Mazo GZ, Benedetti TRB. Adaptação do questionário internacional de atividade física para idosos. Rev Bras Cineantropom Desempenho Hum. 2010;12(6):480-4. https://doi.org/10.5007/1980-0037.2010v12n6p480

13. Wells KF, Dillon EK. The sit and reach: a test of back and leg flexibility. Res Q. 1952;23(1):115-8.

14. Guedes DP, Guedes JER. Crescimento e desempenho motor em escolares do município de Londrina, Paraná, Brasil. Cad Saúde Pública. 1993;9(suppl a):S58-S70. https://doi.org/10.1590/s0102-311x1993000500007

15. Guedes DP, Barbanti VJ. Desempenho motor em crianças e adolescentes. Rev Paul Educ Fís. 1995;9(1):37-50.

16. Achour Júnior A. Avaliando a flexibilidade: manual de instruções. Londrina: Midiograf; 1997.

17. Gorla JI. Educação física especial: testes. Rolândia: Physical-Fisio; 1997. 
18. Achour Júnior A. Flexibilidade e alongamento: saúde e bem-estar. Barueri: Manole; 2004.

19. Woellner SS, Araujo AGS, Martins JS. Protocolos de equilíbrio e quedas em idosos. Neurociências. 2014;10(2):104-17.

20. Dias RG, Streit IA, Sandreschi PF, Benedetti TRB, Mazo GZ. Diferenças nos aspectos cognitivos entre idosos praticantes e não praticantes de exercício físico. J Bras Psiquiatr. 2014;63(4):326-31. https://doi.org/10.1590/00472085000000041

21. Leite MT, Winck MT, Hildebrandt LM, Kirchner RM, Silva LAA. Qualidade de vida e nível cognitivo de pessoas idosas participantes de grupos de convivência. Rev Bras Geriatr Gerontol. 2012;15(3):481-92. https://doi. org/10.1590/s1809-98232012000300009

22. Ruzene JRS, Navega MT. Avaliação do equilíbrio, mobilidade e flexibilidade em idosas ativas e sedentárias. Rev Bras Geriatr Gerontol. 2014;17(4):785-93. https://doi. org/10.1590/1809-9823.2014.13105

23. Matsudo SM, Matsudo VKR, Barros Neto TL. Efeitos benéficos da atividade física na aptidão física e saúde mental durante o processo de envelhecimento. Rev Bras Ativ Fís Saúde. 2000;5(2):60-76. https://doi.org/10.12820/ rbafs.v. 1 n2p5-14

24. Vila CP, Silva MEM, Simas JPN, Guimarães ACA, Parcias SR. Aptidão física funcional e nível de atenção em idosas praticantes de exercício físico. Rev Bras Geriatr Gerontol. 2013;16(2):355-64. https://doi.org/10.1590/s1809. 98232013000200015

25. Rosa AL. A flexibilidade em indivíduos idosos. REI. 2012; $7(15): 1-15$.

26. Bankoff ADP, Campelo TS, Ciol P, Zamai CA. Postura e equilíbrio corporal: um estudo das relações existentes. Mov Percepção. 2006;6(9):55-70.

27. Guedes DP, Guedes JERP. Atividade física, aptidão física e saúde. RBAFS. 1995;1(1):18-35.

28. Santos CS, Cerchiari EAN, Alvarenga MRM, Faccenda O, Oliveira MAC. Avaliação da confiabilidade do mini-exame do estado mental em idosos e associação com variáveis sociodemográficas. Cogitare Enferm. 2010;15(3):406-12. https://doi.org/10.5380/ce.v15i3.18879

29. Lira M, Santos LCCS. Correlação entre função cognitiva e capacidade funcional nos indivíduos com doença de Alzheimer. Cad Pós-Grad Distúrb Desenvolv. 2012; 12 (2):36-45. https://doi.org/10.11606/d.5.2006.tde21112006-174846

30. Diniz BSO, Volpe FM, Tavares AR. Nível educacional e idade no desempenho no Miniexame do Estado Mental em idosos residentes na comunidade. Rev Psiquiatr Clín. 2007;34(1):13-7. https://doi.org/10.1590/s0101. 60832007000100002

31. Fechine BRA, Trompieri N. O processo de envelhecimento: as principais alterações que acontecem com o idoso com o passar dos anos. InterSciencePlace. 2012;1(20):106-32. https://doi.org/10.6020/1679-9844/2007
32. Albino J, Freitas CG, Martins VMS, Kanegusuku H, Roque TP, Bartholomeu T, Forjaz CLM. Tabelas de classificação da aptidão física para frequentadores de parques públicos. Rev Bras Med Esporte. 2010;16(5): 373-7. https://doi.org/10.1590/s1517-86922010000500011

\section{AUTHORS:}

JÉNIFER CAROLINA FERNANDES

Graduada, Universidade Estadual do Oeste do Paraná, Departamento de Educação Física, Marechal Cândido Rondon, PR, Brasil.

E-mail: jheni_13fer@hotmail.com

Orcid: http://orcid.org/0000-0001-6489-5016

ROBSON RUIZ OLIVOTO

Doutor, Universidade Estadual do Oeste do Paraná, Departamento de Educação Física, Marechal Cândido Rondon, PR, Brasil.

E-mail:robson_olivoto@unioeste.br

Orcid: http://orcid.org/0000-0001-6233-6894 